\title{
Establishment of proteome reference maps for somatic and zygotic embryos of Cyclamen persicum Mill.
}

\author{
Christina Rode ${ }^{1 凶}$, Hans-Peter Braun ${ }^{1}$, Sébastien Gallien, ${ }^{2}$ Dimitri Heintz ${ }^{3}$, Alain Van \\ Dorsselaer ${ }^{2}$, Traud Winkelmann ${ }^{4}$ \\ ${ }^{1}$ Institute of Plant Genetics, Leibniz University Hannover, Herrenhaeuser Str. 2, D-30419 \\ Hannover, Germany \\ ${ }^{2}$ Laboratoire de Spectrométrie de Masse Bio-Organique, IPHC-DSA, Université de \\ Strasbourg, CNRS, UMR7178; 25 rue Becquerel, F-67087 Strasbourg, France \\ ${ }^{3}$ Institut de Biologie Moléculaire des Plantes (IBMP) CNRS-UPR2357, Université de \\ Strasbourg, F-67083 Strasbourg, France \\ ${ }^{4}$ Institute of Floriculture and Woody Plant Science, Leibniz University Hannover, \\ Herrenhaeuser Str. 2, D-30419 Hannover, Germany \\ 『rode@genetik.uni-hannover.de
}

Keywords: Differential gel electrophoresis, Embryogenesis, Mass spectrometry, Protein identification, Two-dimensional polyacrylamide gel electrophoresis

Abbreviations: 2D: Two-dimensional, DIGE: Differential gel electrophoresis, EST: Expressed sequence tag, IEF-SDS PAGE: Isoelectric focusing - Sodium dodecyl sulfate Polyacrylamide gel electrophoresis, LC: Liquid chromatography, MALDI- TOF: Matrix Assisted Laser Desorption/Ionisation, MS: Mass spectrometry

\begin{abstract}
Comprehensive proteomic characterizations were performed aiming to create proteome reference maps for somatic and zygotic embryos of Cyclamen persicum. Separation by two dimensional isoelectric focusing - sodium dodecyl sulfate polyacrylamide gel electrophoresis led to a resolution of more than 800 protein spots for each tissue. Approximately $\mathbf{7 0 \%}$ of the spots likewise appeared in both zygotic and in somatic embryo's protein fractions. However, differential gel electrophoresis analyses revealed pronounced differences in abundances for the majority of proteins present in both tissues. MS analyses for 300 reproducible spots in total (263 of the zygotic embryos' protein fraction and 37 spots appearing specifically in the somatic embryos' proteome) led to identification of 261 proteins, 35 of which were specific or highly abundant in gels of the somatic embryo's tissue. Most identified proteins were found to be involved in glycolysis or gluconeogenesis and stress response pathways.
\end{abstract}

\section{INTRODUCTION}

Cyclamen persicum Mill. is a popular ornamental crop with a high economic relevance. For this species somatic embryogenesis has been shown to be an efficient vegetative propagation system (Wicart et al. 1984; Kiviharju et al., 1992; Schwenkel and Winkelmann, 1998). In order to overcome problems due to physiological disorders, the aim of our research is to produce somatic embryos which mimic their zygotic counterparts in protein pattern composition as much as possible. Moreover, the physiological details of somatic and zygotic embryogenesis remain unclear in Cyclamen up to now. Initial analyses comparing the proteomes of somatic and zygotic embryos have been performed previously (Winkelmann et al., 2006) resolving over 200 protein spots per gel. Approximately $70 \%$ of the proteins 
expressed in zygotic embryos also were present in somatic embryos in comparable abundances. MS analyses of 83 spots led to the identification of 20 proteins of both tissues and additionally fife endosperm proteins (Winkelmann et al., 2006). Lyngved et al. (2008) analysed an embryogenic and one non embryogenic cell line of $C$. persicum, using a comprehensive 2D DIGE approach. Of the 1200 detected protein spots 108 were found to be more abundant in the embryogenic cells, and 97 proteins in the non-embryogenic cells. MS analyses via MALDI-TOF led to the identification of 128 proteins including unique proteins for both tissues. In addition a transcriptome analysis was reported (Rensing et al., 2005), resulting in an EST database with 2083 sequences (www.cyclamen-est.de). The EST database constitutes the basis of molecular characterisation of $C$. persicum, since very few genomic Cyclamen specific sequences are available in public databases up to now. Based on the analyses of Winkelmann et al. (2006) further comprehensive and standardized proteomic characterizations were performed as presented in the current study, aiming to create proteome reference maps for somatic and zygotic embryos and to elucidate the physiology of embryo development in C. persicum.

\section{MATERIALS AND METHODS}

Somatic and zygotic embryos were derived from the commercial $F_{1}$ cultivar 'Maxora Light Purple' of $C$. persicum bred by the company Varinova (Berkel en Rodenrijs, Netherlands). Somatic embryos were produced via somatic embryogenesis: Calluses were initiated from unpollinated ovules placed on modified Murashige and Skoog medium (Murashige and Skoog, 1962) containing $9.05 \mu \mathrm{M}$ 2,4-D and $3.94 \mu \mathrm{M} 2 \mathrm{iP}$ as described by Schwenkel and Winkelmann (1998). After 8 weeks of culture, embryogenic calluses emerged which were used to start the suspension culture in liquid Murashige and Skoog media (Winkelmann et al., 1998). A 500-1000 $\mu \mathrm{m}$ fraction of this suspension was plated on hormone-free Murashige and Skoog medium (Murashige and Skoog, 1962) and torpedoshaped embryos were harvested after 4 weeks of culture. Zygotic embryos were prepared out of the seeds 11 weeks after self pollination. Both tissues were directly frozen in liquid nitrogen after harvesting and pulverized in a bead mill.

To find a protein extraction method which allows very efficient protein purification and at the same time is compatible with high-resolution protein separation, four protocols were tested with 10 - $150 \mathrm{mg}$ somatic embryo tissue of $C$. persicum and replicated at least 3 times: A rapid protocol without precipitation as described by Gallardo et al. (2002), a protocol utilising TCA precipitation in combination with acetone (Damervahl et al.,1986), an extraction method using phenol combined with a ammonium acetate in methanol precipitation (Hurkman and Tanaka, 1986, modified by Colditz et al., 2004) and a protocol described by Corcke and Roberts (1997) where samples are boiled in Laemmli buffer (Laemmli, 1970) and proteins subsequently precipitated with acetone. Complete protein extracts were loaded on gels and separated by their isoelectric point and their size via IEF-SDS PAGE as described by Winkelmann et al. (2006), but using immobiline dry strip gels with a $\mathrm{pH}$ gradient of 3-11. For each tissue 4 biological and technical replications were performed. Gels were scanned and quantitatively and qualitatively evaluated using ImageMaster 2D Platinum 6.0 software (GE healthcare, Munich, Germany). The 263 most abundant protein spots were picked manually from a 2D IEF-SDS gel of zygotic embryos proteome extract using GelPal Protein Excision System (Genetix, Queensway, U.K). In addition, 37 spots were picked from a gel of somatic embryos' proteome extract, which are specific or highly abundant within this tissue. In situ digestion of protein spots and nano-LC-MS/MS analyses as well as protein database searches were performed according to Fuehrs et al. (2009).

For the 2D DIGE approach zygotic and somatic protein fractions were labelled each with CyDye fluorophors, that are spectrally resolvable. Protein fractions, each $100 \mu \mathrm{g}$, were 
pooled, loaded on one gel and separated via IEF-SDS PAGE as described above. Subsequently, 2D gels were scanned at dye specific wavelengths.

\section{RESULTS AND DISCUSSION}

Optimal protein purification and resolution on IEF-SDS gel was achieved using the modified Hurkman and Tanaka (1986) protocol with $80 \mathrm{mg}$ fresh tissue. Separation by two dimensional IEF-SDS gel electrophoresis led to a resolution of more than 800 protein spots for each tissue. Gels representative for each tissue are shown in Figure 1. Consistent to the initial analysis (Winkelmann et al., 2006), approximately $70 \%$ of the spots likewise appeared in both zygotic and in somatic protein fractions. However, DIGE analysis revealed extremely high alterations in abundances for the majority of proteins present in both tissues (data not shown). Protein spot groups and single protein spots highly abundant or specific for both tissues are marked by white circles in Figure 1. Group a and b were observed to be highly abundant/specific in the somatic embryos, while groups $\mathrm{c}, \mathrm{d}, \mathrm{e}, \mathrm{f}$ and protein spot $\mathrm{g}$ were highly abundant/specific for the zygotic embryos' proteome. Mass spectrometry analyses via nano-LC MS/MS for a total of 300 highly abundant and reproducible spots of both protein fractions led to the identification of 261 protein spots whereof 35 were highly abundant/specific for the somatic tissue. In total, 90 of the identified spots reflected non redundant proteins, most of them involved in glycolysis/gluconeogenesis and stress response pathways. In comparison with previous proteomic analysis by Winkelmann et al. (2006) differential protein resolution was increased to at least 800 spots per gel. This was mainly the result of ten times higher tissue input and a protein extraction method with a precipitation step and phenol application (Hurkman and Tanaka, 1986) leading to raised protein concentrations and more purified protein fractions. Thus, MS based protein identification rate could be increased to $87 \%$ of picked protein spots (261 out of 300 ) also due to the fact that the number of available sequences in public databases has been increased. The protein identification rate of $87 \%$ is supposed to be quite high, even though C. persicum has no close relatives within the databases of sequenced plants. This fact illustrates that protein identification in non-model organisms recently became more and more successful.

\section{CONCLUSIONS}

Here, we have applied a 2D differential gel electrophoresis approach to characterise and elucidate the proteins involved in somatic and zygotic embryogenesis in Cyclamen persicum. High protein resolution combined with nano-LC MS/MS analyses led to the identification of the majority of the highly abundant spots in both tissues.

Currently, identified proteins are clustered according to their physiological relevance and a digital proteome reference map is created and will be made publicly available soon.

\section{ACKNOWLEDGMENTS}

The authors would like to thank the DFG (Deutsche Forschungsgemeinschaft German Research Founding) for financial support and Dr. Frank Colditz for critically reading the manuscript. 


\section{Literature Cited}

Colditz, F., Nyamsuren, O., Niehaus, H., Eubel, H., Braun, H.-P. and Krajinski, F. 2004. Proteomic approach: Identification of Medicago truncatula proteins induced in roots after infection with the pathogenic oomycete Aphanomyces euteiches. Plant. Mol. Biol. 55: 109-120.

Corke, F. M. K. and Roberts, K. 1996. Large changes in the population of cell wall proteins accompany the shift to cell elongation. J. Exp. Bot. 48: 971-977.

Damerval, C., De Vienne, D., Zivy, M. and Thiellement, H. 1986. Technical improvements in two-dimensional electrophoresis increase the level of genetic variation detected in wheat-seedling proteins. Electrophoresis. 7: 52-54.

Fuehrs, H., Goetze, S., Specht, A., Erban, A., Gallien, S., Heintz, D., Van Dorsselaer, A., Kopka, J., Braun, H. P., Horst, W. J. 2009. Characterization of leaf apoplastic peroxidases and metabolites in Vigna unguiculata in response to toxic manganese supply and silicon J. Exp. Bot. 60:1663-1678.

Gallardo, K., Job, C., Groot, S. P. C., Puype, M., Demol, H., Vandekerckhove, J. and Job, D. 2002. Proteomics of Arabidopsis seed germination: a comparative study of wild-type and gibberelline deficient seeds. Plant. Physiol. 129: 823-837.

Hurkman, W. J. and Tanaka C. K. 1986. Solubilization of plant membrane proteins for analysis by two-dimensional gel electrophoresis. Plant Physiol. 81: 802-806.

Kiviharju, E., Tuominen, U., Törmälä, T. 1992. The effect of explant material on somatic embryogenesis of Cyclamen persicum Mill. Plant Cell Tissue Organ Cult. 28: 187-194

Laemmli, U. K. 1970. Cleavage of structural proteins during the assembly of the head of bacteriophage T4. Nature. 227: 680-685.

Lyngved, R., Renaut, J., Hausman, J.-F., Iversen, T.-H. and Hvoslef-Eide, A. K. 2008. Embryo-specific proteins in Cyclamen persicum analyzed with 2-D DIGE. J. Plant Growth Regul. 27: 353-369.

Murashige, T. and Skoog, F. 1962. A revised medium for rapid growth and bioassays with tobacco tissue cultures. Physiol. Plant. 15: 473-497.

Rensing, S. A., Lang, D., Schumann, E., Reski, R. and Hohe, A. 2005. EST sequencing from embryogenic Cyclamen persicum cell cultures identifies a high proportion of transcripts homologous to plant genes involved in somatic embryogenesis. J. Plant Growth Regul. 24: 102-115.

Schwenkel, H.-G. and Winkelmann T. 1998. Plant regeneration via somatic embryogenesis from ovules of Cyclamen persicum Mill. Plant Tissue Culture and Biotechnology 4: 28-34.

Wicart, G., Mouras, A., Lutz, A. 1984. Histological study of organogenesis and embryogenesis in Cyclamen persicum tissue cultures: evidence for a single organogenetic pattern. Protoplasma 119: 159-167.

Winkelmann, T., Hohe, A., Schwenkel, H.G. 1998. Establishing embryogenic suspension cultures in Cyclamen persicum 'Purple Flamed'. Adv. Hortic. Sci. 12: 25-30.

Winkelmann, T., Heintz D., Van Dorsselaer, A., Serek, M., and Braun, H.-P. 2006. Proteomic analyses of somatic and zygotic embryos of Cyclamen persicum Mill. reveal new insights into seed and germination physiology. Planta. 224: 508-519. 

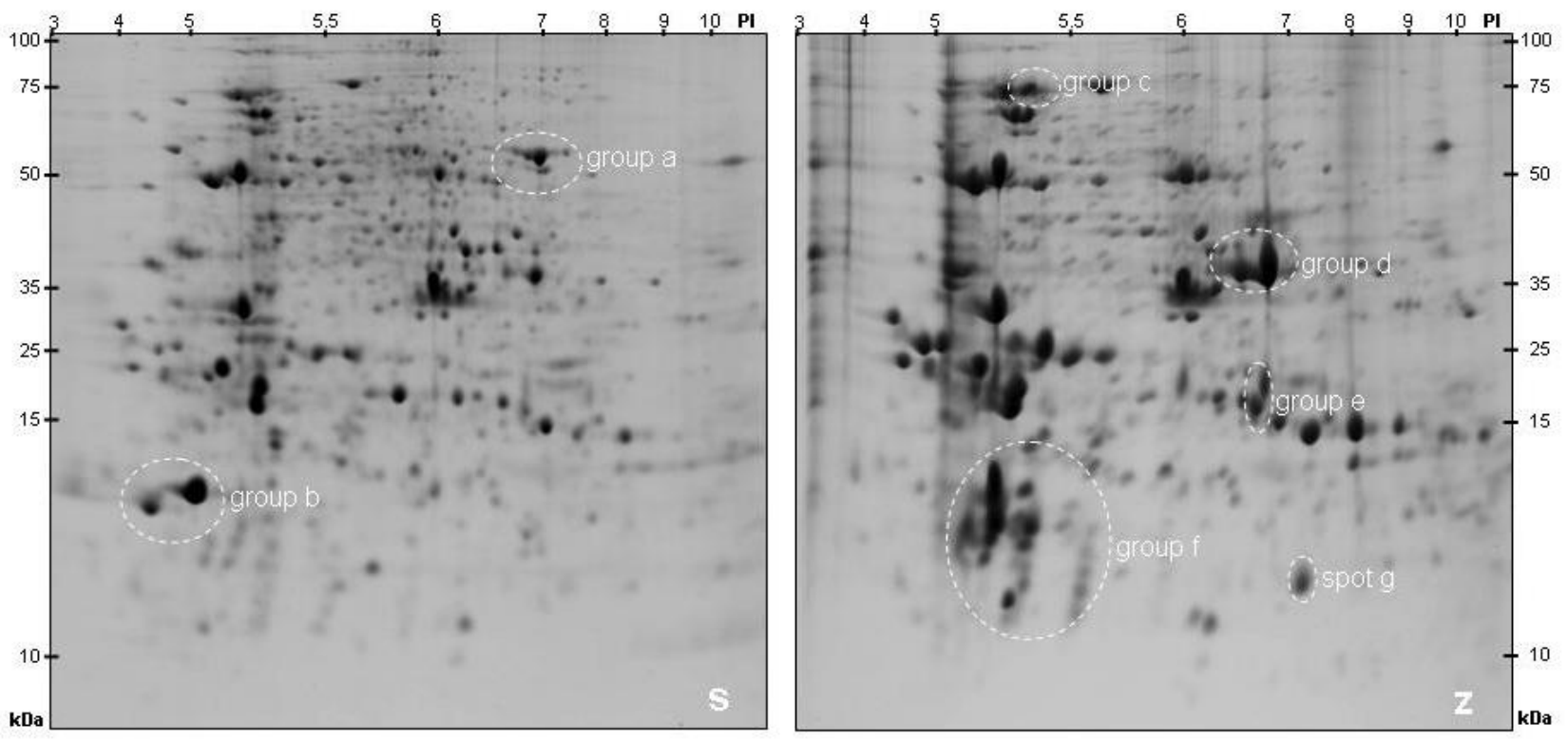

Fig. 1. IEF-SDS PAGE of somatic (s) and zygotic (z) embryo's proteome of Cyclamen persicum

Total protein from zygotic and somatic embryos were loaded on the gels. Proteins were separated in the first gel dimension according to their isolelectric points ( $\mathrm{pI}$ range of 3 to 11) and in the second dimension on the basis of their molecular masses $(10-100 \mathrm{kDa}$ ). Regions with tissue specific or highly abundant protein spots are marked by white circles. 